\title{
Allografts with titanium cage in the treatment of tuberculous spondylitis: at least 3-year follow-up study
}

\author{
Yanjun Wang \\ graduate school of Tianjin Medical University \\ Hong Zhang ( $\square$ wangyj1973@126.com ) \\ PLA Army General Hospital
}

\section{Research}

Keywords: Tuberculous spondylitis, Allograft, Titanium cage, Surgical intervention

Posted Date: July 22nd, 2020

DOI: https://doi.org/10.21203/rs.3.rs-39556/v1

License: @ (1) This work is licensed under a Creative Commons Attribution 4.0 International License. Read Full License 


\section{Abstract}

Background Anterior radical debridement and spinal fusion with instrumentation have been advocated in treating the spinal tuberculosis patients. But there are few long-term studies on the efficacy of fresh-frozen allografts with titanium cage in reconstruction of the anterior spinal column after debridement and decompression for tuberculosis. The purpose of this study was to evaluate the efficacy of anterior allografts with titanium cage which were stabilized with anterior or posterior instrumentation at least 3-year follow-up study.

Methods A retrospective analysis of 133 patients were treated between January 2002 and December 2012. There were 71 men and 62 women. Those patients were evaluated according to clinical and radiographical record for a minimum of 3 year, including deformity correction, pain and neurologic status. The kyphosis deformity was measured on lateral radiographs according the angle between the superior endplate of the first upper uninvolved vertebra and the first lower uninvolved vertebra. In the lumbosacral region, the kyphosis angle was measured by two lines along the posterior border of the first normal vertebra above the level of the lesion and posterior margin of sacrum on lateral radiographs. Clinical evaluation of back pain was evaluated and documented according to VAS pain score. Patients with neural deficits were graded according to the ASIA scale.

Results The preoperative kyphosis deformity improved in all patients postoperatively from a mean of $20.8^{\circ}$ (range, $12^{\circ}-35^{\circ}$ ) to $3.9^{\circ}$ (range, $\left.2^{\circ}-7^{\circ}\right)$, with the average deformity correction of $16.1^{\circ}(t=38.59, p<0.0001)$. The earliest radiologic evidence of allograft incorporation was identified between 18 and 24 months after surgery, and bony fusion was seen in all patients at final follow-up. We put forward our criteria to classify allograft incorporation at the last follow-up. Grade 149 patients; Grade 273 patients; Grade 311 patients; Grade 4 no patient. The average VAS pain score was 7.7 (range, 6-9) preoperatively, and decreased to 1.4 (range, $1-3$ ) 3 months after surgery $(t=97.88, p<0.0001$ vs. initial). Of the 44 patients who developed neural deficits before their operations, 41 patients improved according to the ASIA AIS. 3 patients did not have substantial improvement and remained Grade AIS B.

Conclusions Radical debridement followed by allograft with titanium cage in reconstruction of the anterior spinal column offers very satisfactory results in patients with tuberculous spondylitis. It provides immediate stability, offers lasting kyphosis correction, bypasses the donor site morbidity and difficulties involved in obtaining structural autograft.

\section{Background}

Musculoskeletal tuberculosis accounts for approximately $10 \%$ of all extrapulmonary tuberculosis cases [1] and spinal tuberculosis comprises up to $50 \%$ of musculoskeletal tuberculosis [2]. Approximately $98 \%$ of spinal tuberculosis affects the anterior column of spine [3]. These patients often develop collapse of vertebrae because of tuberculous destruction, resulting in varying degrees of kyphosis, and 10\% 47\% develop neurologic deficits [4]. Antituberculous chemotherapy and immobilization lead to a favorable outcome in most of the patients by preventing abscess formation and neurologic deterioration [5]. Surgical intervention with radical debridement and stabilization is sometimes considered for the patients with severe pain from abscess, neurologic impairment from spinal cord compression, significant bone destruction, progressive kyphotic deformity or instability and antituberculous-resistant or recurrence $[2,6]$. Eradication of infection is the primary aim of surgical treatment in spinal tuberculosis, with the prevention or correction of spinal deformity and recovery from neurological deficits [7]. Anterior radical debridement and spinal fusion with instrumentation have been advocated in treating the patients with spinal tuberculosis [8, 9]. Radical debridement of the compromised anterior column that has lost its structural integrity requires reconstruction for prevention or correction of spinal deformity [10-12]. There are few long-term studies on the efficacy of fresh-frozen allografts with titanium cage in reconstruction of the anterior spinal column after debridement and decompression for tuberculosis [12-14]. In the current study, the efficacy of anterior allografts with titanium cage, which were stabilized with anterior or posterior instrumentation, were evaluated 3 to 8 years after anterior radical debridement and spinal fusion for spinal tuberculosis.

\section{Materials And Methods}

A retrospective analysis of 133 patients with tuberculous spondylitis of the thoracic and lumbar spine treated by anterior radical debridement and spinal fusion using allografts with titanium cage was performed to evaluate the incorporation of allografts. Approval to conduct this study was obtained from our institutional review board, and informed consent was obtained from each patient. These patients were treated between January 2002 and December 2012 in the Department of Spine Surgery, The Second Hospital of Tangshan, China. The main indication for surgery was severe pain from abscess, neurologic impairment from spinal cord compression, significant bone destruction, progressive kyphotic deformity or instability and antituberculous-resistant or recurrence.

There were 71 men and 62 women. Mean ( \pm Standard Deviation) age of this series was $46.9 \pm 12.3$ years (range, 20-69 years). Patients were evaluated according to clinical and radiographical record for a minimum of 3 year, including deformity correction, pain, and neurologic status. 
The mean follow-up period was 46.7 months (range, 36-57 months). The demographic and diagnostic characteristics of patients were listed in the Table 1. 
Table 1

Summary of clinical date in the serials

\begin{tabular}{|c|c|c|c|c|c|c|c|c|c|c|c|c|c|c|}
\hline \multirow{2}{*}{$\begin{array}{l}\text { Case } \\
\text { No. }\end{array}$} & \multirow{2}{*}{$\begin{array}{l}\text { Age(Y) } \\
\text { /SeX }\end{array}$} & \multirow[t]{2}{*}{ Level } & \multirow{2}{*}{$\begin{array}{l}\text { Duration of } \\
\text { disease(mo) }\end{array}$} & \multirow{2}{*}{$\begin{array}{l}\text { Surgical } \\
\text { procedure }\end{array}$} & \multicolumn{3}{|c|}{ Kyphotic angle } & \multicolumn{3}{|c|}{ VAS pain score } & \multicolumn{2}{|c|}{$\begin{array}{l}\text { Neurologic } \\
\text { status(ASIA) }\end{array}$} & \multirow{2}{*}{$\begin{array}{l}\text { Fusion } \\
\text { grade }\end{array}$} & \multirow{2}{*}{$\begin{array}{l}\text { Follow- } \\
\text { up } \\
\text { (months) }\end{array}$} \\
\hline & & & & & $\begin{array}{l}\text { Pre- } \\
\text { op }\end{array}$ & $\begin{array}{l}\text { Post- } \\
\text { op }\end{array}$ & FFU & $\begin{array}{l}\text { Pre- } \\
\text { op }\end{array}$ & $\begin{array}{l}\text { Post- } \\
\text { op }\end{array}$ & FFU & $\begin{array}{l}\text { Pre- } \\
\text { op }\end{array}$ & FFU & & \\
\hline 1 & $52 / F$ & $\begin{array}{l}\text { L3] } \\
\text { L4 }\end{array}$ & 14 & CAPP & 27 & 3 & 3 & 7 & 1 & 0 & $E$ & $E$ & 2 & 56 \\
\hline 2 & $61 / M$ & $\begin{array}{l}\text { L2] } \\
\text { L3 }\end{array}$ & 11 & AP & 21 & 5 & 6 & 7 & 1 & 0 & $E$ & $E$ & 2 & 57 \\
\hline 3 & $39 / \mathrm{M}$ & $\begin{array}{l}\text { L4! } \\
\text { L5 }\end{array}$ & 4 & CAPP & 19 & 3 & 4 & 7 & 1 & 0 & $E$ & $E$ & 2 & 52 \\
\hline 4 & $44 / F$ & $\begin{array}{l}\mathrm{L} 4 \square \\
\mathrm{L} 5\end{array}$ & 9 & CAPP & 19 & 3 & 3 & 6 & 1 & 0 & $E$ & $E$ & 3 & 36 \\
\hline 5 & $25 / F$ & $\begin{array}{l}\mathrm{L} 4 \sim \\
\mathrm{S} 1\end{array}$ & 12 & CAPP & 28 & 5 & 6 & 9 & 3 & 2 & $E$ & $E$ & 3 & 46 \\
\hline 6 & $39 / F$ & L5 & 4 & CAPP & 23 & 2 & 2 & 9 & 3 & 2 & $E$ & $E$ & 1 & 41 \\
\hline 7 & $52 / \mathrm{M}$ & $\begin{array}{l}\text { L5! } \\
\text { S1 }\end{array}$ & 4 & CAPP & 22 & 2 & 2 & 8 & 1 & 1 & $E$ & $E$ & 2 & 52 \\
\hline 8 & $46 / \mathrm{M}$ & $\begin{array}{l}\text { L3! } \\
\text { L4 }\end{array}$ & 4 & CAPP & 24 & 3 & 4 & 8 & 2 & 1 & $E$ & $E$ & 2 & 44 \\
\hline 9 & $59 / F$ & $\begin{array}{l}\text { L5! } \\
\text { S1 }\end{array}$ & 9 & CAPP & 23 & 2 & 2 & 8 & 3 & 2 & $E$ & $E$ & 3 & 57 \\
\hline 10 & $52 / F$ & $\begin{array}{l}\text { L4! } \\
\text { L5 }\end{array}$ & 6 & CAPP & 16 & 2 & 2 & 7 & 1 & 0 & $E$ & $E$ & 2 & 41 \\
\hline 11 & $38 / F$ & $\begin{array}{l}\text { L10 } \\
\text { L2 }\end{array}$ & 5 & AP & 18 & 3 & 4 & 7 & 1 & 0 & $E$ & $E$ & 1 & 49 \\
\hline 12 & $51 / F$ & $\begin{array}{l}\text { L3] } \\
\text { L4 }\end{array}$ & 15 & CAPP & 20 & 5 & 6 & 8 & 2 & 0 & $E$ & $E$ & 2 & 44 \\
\hline 13 & $59 / F$ & L5 & 11 & CAPP & 21 & 5 & 7 & 7 & 1 & 0 & $E$ & $E$ & 2 & 47 \\
\hline 14 & $48 / \mathrm{M}$ & $\begin{array}{l}\text { L3! } \\
\text { L4 }\end{array}$ & 4 & CAPP & 22 & 3 & 3 & 7 & 1 & 0 & $E$ & $E$ & 1 & 51 \\
\hline 15 & $43 / F$ & L4 & 10 & CAPP & 23 & 3 & 4 & 7 & 1 & 0 & $E$ & $E$ & 1 & 42 \\
\hline 16 & $49 / \mathrm{M}$ & $\begin{array}{l}\text { T12 } \\
\text { L1 }\end{array}$ & 11 & AP & 18 & 6 & 6 & 7 & 1 & 0 & $D$ & $E$ & 2 & 43 \\
\hline 17 & $42 / F$ & $\begin{array}{l}\text { L10 } \\
\text { L2 }\end{array}$ & 15 & AP & 20 & 5 & 6 & 7 & 1 & 0 & $E$ & $E$ & 2 & 39 \\
\hline 18 & $27 / M$ & $\begin{array}{l}\text { T12 } \\
\sim \text { L2 }\end{array}$ & 8 & CAPP & 31 & 6 & 6 & 9 & 1 & 1 & $E$ & $E$ & 2 & 42 \\
\hline 19 & $34 / F$ & $\begin{array}{l}\text { L10 } \\
\text { L2 }\end{array}$ & 12 & AP & 19 & 6 & 7 & 8 & 1 & 0 & $E$ & $E$ & 1 & 56 \\
\hline 20 & $62 / M$ & $\begin{array}{l}\text { L2 } \\
\text { L3 }\end{array}$ & 6 & AP & 14 & 2 & 4 & 7 & 1 & 0 & $E$ & $E$ & 2 & 46 \\
\hline 21 & $61 / \mathrm{M}$ & $\begin{array}{l}\text { L2] } \\
\text { L3 }\end{array}$ & 15 & AP & 20 & 6 & 6 & 8 & 2 & 1 & $E$ & $E$ & 2 & 43 \\
\hline 22 & $56 / \mathrm{M}$ & $\begin{array}{l}\text { T6 } \\
\text { T9 }\end{array}$ & 15 & CAPP & 29 & 6 & 7 & 7 & 1 & 0 & D & $E$ & 2 & 45 \\
\hline 23 & $68 / F$ & $\begin{array}{l}\mathrm{L} 10 \\
\mathrm{~L} 2\end{array}$ & 14 & AP & 13 & 2 & 3 & 9 & 3 & 2 & D & $E$ & 3 & 38 \\
\hline 24 & $42 / F$ & $\begin{array}{l}\text { T12 } \\
\text { L1 }\end{array}$ & 3 & AP & 12 & 2 & 5 & 7 & 2 & 1 & D & $E$ & 1 & 55 \\
\hline
\end{tabular}




\begin{tabular}{|c|c|c|c|c|c|c|c|c|c|c|c|c|c|c|}
\hline \multirow{2}{*}{$\begin{array}{l}\text { Case } \\
\text { No. }\end{array}$} & \multirow{2}{*}{$\begin{array}{l}\operatorname{Age}(Y) \\
/ \text { Sex }\end{array}$} & \multirow[t]{2}{*}{ Level } & \multirow{2}{*}{$\begin{array}{l}\text { Duration of } \\
\text { disease(mo) }\end{array}$} & \multirow{2}{*}{$\begin{array}{l}\text { Surgical } \\
\text { procedure }\end{array}$} & \multicolumn{3}{|c|}{ Kyphotic angle } & \multicolumn{3}{|c|}{ VAS pain score } & \multicolumn{2}{|c|}{$\begin{array}{l}\text { Neurologic } \\
\text { status(ASIA) }\end{array}$} & \multirow{2}{*}{$\begin{array}{l}\text { Fusion } \\
\text { grade }\end{array}$} & \multirow{2}{*}{$\begin{array}{l}\text { Follow- } \\
\text { up } \\
\text { (months) }\end{array}$} \\
\hline & & & & & $\begin{array}{l}\text { Pre- } \\
\text { op }\end{array}$ & $\begin{array}{l}\text { Post- } \\
\text { op }\end{array}$ & FFU & $\begin{array}{l}\text { Pre- } \\
\text { op }\end{array}$ & $\begin{array}{l}\text { Post- } \\
\text { op }\end{array}$ & FFU & $\begin{array}{l}\text { Pre- } \\
\text { op }\end{array}$ & FFU & & \\
\hline 25 & $32 / F$ & $\begin{array}{l}\text { T100 } \\
\text { T11 }\end{array}$ & 7 & AP & 20 & 6 & 6 & 6 & 1 & 0 & C & D & 1 & 46 \\
\hline 26 & $52 / \mathrm{M}$ & $\begin{array}{l}\text { L4! } \\
\text { L5 }\end{array}$ & 7 & CAPP & 23 & 2 & 2 & 9 & 2 & 1 & E & E & 2 & 45 \\
\hline 27 & $47 / M$ & $\begin{array}{l}\mathrm{L} 2 \sim \\
\mathrm{L} 4\end{array}$ & 5 & CAPP & 23 & 5 & 6 & 8 & 1 & 0 & E & E & 2 & 53 \\
\hline 28 & $28 / F$ & $\begin{array}{l}\mathrm{L} 2 \square \\
\mathrm{L} 3\end{array}$ & 4 & AP & 18 & 5 & 5 & 7 & 1 & 0 & E & E & 1 & 47 \\
\hline 29 & $46 / F$ & T10 & 5 & $\mathrm{AP}$ & 15 & 5 & 5 & 6 & 1 & 0 & D & E & 1 & 57 \\
\hline 30 & $46 / \mathrm{M}$ & $\begin{array}{l}\mathrm{L} 2 \sim \\
\mathrm{L} 5\end{array}$ & 13 & CAPP & 25 & 7 & 8 & 8 & 2 & 1 & E & E & 3 & 51 \\
\hline 31 & $29 / F$ & $\begin{array}{l}\mathrm{L} 2 \square \\
\mathrm{L} 3\end{array}$ & 12 & AP & 12 & 2 & 4 & 8 & 1 & 1 & E & E & 2 & 36 \\
\hline 32 & $53 / \mathrm{M}$ & $\begin{array}{l}\text { L40 } \\
\text { L5 }\end{array}$ & 8 & CAPP & 22 & 2 & 3 & 7 & 1 & 0 & E & E & 2 & 39 \\
\hline 33 & $61 / \mathrm{M}$ & $\mathrm{T} 12$ & 8 & $\mathrm{AP}$ & 15 & 2 & 2 & 8 & 2 & 1 & D & E & 2 & 54 \\
\hline 34 & $48 / \mathrm{M}$ & $\begin{array}{l}\mathrm{L} 10 \\
\mathrm{~L} 2\end{array}$ & 6 & $\mathrm{AP}$ & 18 & 5 & 5 & 9 & 2 & 1 & E & E & 2 & 42 \\
\hline 35 & $32 / \mathrm{M}$ & $\begin{array}{l}\text { L40 } \\
\text { L5 }\end{array}$ & 9 & CAPP & 21 & 3 & 4 & 7 & 1 & 0 & $E$ & $\mathrm{E}$ & 1 & 54 \\
\hline 36 & $45 / \mathrm{M}$ & $\begin{array}{l}\text { L4! } \\
\text { L5 }\end{array}$ & 9 & CAPP & 19 & 4 & 4 & 8 & 1 & 0 & E & $\mathrm{E}$ & 1 & 36 \\
\hline 37 & $59 / F$ & $\begin{array}{l}\text { L5 } \\
\text { S1 }\end{array}$ & 11 & CAPP & 16 & 3 & 3 & 9 & 1 & 0 & E & $\mathrm{E}$ & 2 & 45 \\
\hline 38 & $40 / \mathrm{M}$ & $\begin{array}{l}\mathrm{L} 2 \square \\
\mathrm{L} 3\end{array}$ & 14 & CAPP & 27 & 4 & 6 & 9 & 3 & 2 & E & E & 2 & 41 \\
\hline 39 & $21 / F$ & $\begin{array}{l}\text { T9 } \\
\text { L1 }\end{array}$ & 15 & CAPP & 33 & 3 & 4 & 8 & 1 & 1 & E & E & 3 & 36 \\
\hline 40 & $50 / F$ & $\begin{array}{l}\mathrm{L} 4 \square \\
\mathrm{L} 5\end{array}$ & 4 & CAPP & 21 & 4 & 4 & 7 & 2 & 1 & E & E & 2 & 57 \\
\hline 41 & $52 / \mathrm{M}$ & $\begin{array}{l}\text { T7 } \\
\text { T9 }\end{array}$ & 4 & CAPP & 35 & 5 & 6 & 8 & 1 & 1 & C & D & 2 & 49 \\
\hline 42 & $47 / F$ & $\begin{array}{l}\text { T8 } \\
\text { T10 }\end{array}$ & 6 & CAPP & 28 & 3 & 4 & 8 & 1 & 1 & C & D & 2 & 45 \\
\hline 43 & $29 / \mathrm{M}$ & $\begin{array}{l}\text { T9 } \\
\text { T12 }\end{array}$ & 8 & CAPP & 29 & 7 & 9 & 9 & 2 & 1 & D & $\mathrm{E}$ & 3 & 51 \\
\hline 44 & $46 / F$ & $\begin{array}{l}\text { T90 } \\
\text { T10 }\end{array}$ & 9 & AP & 12 & 3 & 5 & 7 & 1 & 0 & D & E & 2 & 44 \\
\hline 45 & $39 / F$ & $\begin{array}{l}\text { L3ロ } \\
\text { L4 }\end{array}$ & 9 & CAPP & 21 & 3 & 3 & 7 & 1 & 0 & E & E & 1 & 52 \\
\hline 46 & $52 / \mathrm{M}$ & $\begin{array}{l}\text { L4! } \\
\text { L5 }\end{array}$ & 10 & CAPP & 13 & 4 & 4 & 7 & 1 & 0 & E & E & 2 & 47 \\
\hline 47 & $42 / \mathrm{M}$ & $\begin{array}{l}\mathrm{L} 10 \\
\mathrm{~L} 2\end{array}$ & 10 & AP & 13 & 5 & 6 & 7 & 1 & 0 & E & $\mathrm{E}$ & 1 & 36 \\
\hline 48 & $59 / F$ & L2 & 9 & $\mathrm{AP}$ & 20 & 4 & 6 & 7 & 1 & 0 & E & E & 2 & 50 \\
\hline
\end{tabular}




\begin{tabular}{|c|c|c|c|c|c|c|c|c|c|c|c|c|c|c|}
\hline \multirow{2}{*}{$\begin{array}{l}\text { Case } \\
\text { No. }\end{array}$} & \multirow{2}{*}{$\begin{array}{l}\text { Age(Y) } \\
\text { /Sex }\end{array}$} & \multirow[t]{2}{*}{ Level } & \multirow{2}{*}{$\begin{array}{l}\text { Duration of } \\
\text { disease(mo) }\end{array}$} & \multirow{2}{*}{$\begin{array}{l}\text { Surgical } \\
\text { procedure }\end{array}$} & \multicolumn{3}{|c|}{ Kyphotic angle } & \multicolumn{3}{|c|}{ VAS pain score } & \multicolumn{2}{|c|}{$\begin{array}{l}\text { Neurologic } \\
\text { status(ASIA) }\end{array}$} & \multirow{2}{*}{$\begin{array}{l}\text { Fusion } \\
\text { grade }\end{array}$} & \multirow{2}{*}{$\begin{array}{l}\text { Follow- } \\
\text { up } \\
\text { (months) }\end{array}$} \\
\hline & & & & & $\begin{array}{l}\text { Pre- } \\
\text { op }\end{array}$ & $\begin{array}{l}\text { Post- } \\
\text { op }\end{array}$ & FFU & $\begin{array}{l}\text { Pre- } \\
\text { op }\end{array}$ & $\begin{array}{l}\text { Post- } \\
\text { op }\end{array}$ & FFU & $\begin{array}{l}\text { Pre- } \\
\text { op }\end{array}$ & FFU & & \\
\hline 49 & $68 / F$ & $\begin{array}{l}\text { L2" } \\
\text { L3 }\end{array}$ & 8 & AP & 14 & 5 & 5 & 7 & 1 & 0 & $E$ & E & 3 & 41 \\
\hline 50 & $57 / F$ & $\begin{array}{l}\mathrm{L} 10 \\
\mathrm{~L} 2\end{array}$ & 9 & $\mathrm{AP}$ & 19 & 2 & 4 & 8 & 1 & 1 & C & E & 2 & 41 \\
\hline 51 & $49 / \mathrm{M}$ & $\begin{array}{l}\mathrm{L} 3 \square \\
\mathrm{L} 4\end{array}$ & 4 & CAPP & 24 & 5 & 5 & 8 & 1 & 1 & $E$ & E & 1 & 46 \\
\hline 52 & $67 \mathrm{~F}$ & $\begin{array}{l}\text { T110 } \\
\text { T12 }\end{array}$ & 3 & AP & 26 & 4 & 5 & 8 & 1 & 1 & C & D & 3 & 55 \\
\hline 53 & $41 / F$ & $\begin{array}{l}\text { L30 } \\
\text { L4 }\end{array}$ & 4 & CAPP & 21 & 3 & 4 & 7 & 1 & 0 & $\mathrm{E}$ & E & 1 & 43 \\
\hline 54 & $68 / \mathrm{M}$ & $\begin{array}{l}\text { L2" } \\
\text { L3 }\end{array}$ & 11 & AP & 17 & 3 & 5 & 7 & 1 & 0 & $E$ & E & 2 & 44 \\
\hline 55 & $52 / \mathrm{M}$ & $\begin{array}{l}\mathrm{L} 2 \square \\
3 \mathrm{~L}\end{array}$ & 4 & CAPP & 24 & 6 & 6 & 7 & 1 & 0 & $E$ & E & 2 & 54 \\
\hline 56 & $41 / \mathrm{M}$ & $\begin{array}{l}\text { L10 } \\
\text { L2 }\end{array}$ & 11 & AP & 13 & 6 & 7 & 7 & 1 & 0 & C & D & 1 & 56 \\
\hline 57 & $35 / \mathrm{M}$ & $\begin{array}{l}\mathrm{L} 1 \sim \\
\mathrm{L} 3\end{array}$ & 3 & CAPP & 33 & 7 & 8 & 7 & 1 & 0 & $D$ & E & 2 & 37 \\
\hline 58 & $59 / \mathrm{M}$ & $\begin{array}{l}\text { T60 } \\
\text { T7 }\end{array}$ & 14 & AP & 24 & 4 & 4 & 8 & 1 & 0 & $D$ & E & 2 & 48 \\
\hline 59 & $40 / \mathrm{M}$ & $\begin{array}{l}\text { T110 } \\
\text { T12 }\end{array}$ & 4 & AP & 18 & 4 & 6 & 8 & 2 & 1 & $D$ & $E$ & 2 & 41 \\
\hline 60 & $53 / \mathrm{M}$ & $\begin{array}{l}\mathrm{L} 3 \square \\
\mathrm{L} 4\end{array}$ & 10 & CAPP & 24 & 2 & 3 & 7 & 1 & 0 & $E$ & E & 1 & 37 \\
\hline 61 & $29 / F$ & $\begin{array}{l}\text { T100 } \\
\text { T11 }\end{array}$ & 8 & CAPP & 25 & 5 & 5 & 8 & 1 & 1 & $E$ & $E$ & 1 & 44 \\
\hline 62 & $45 / \mathrm{M}$ & $\begin{array}{l}\text { T7 } \\
\text { T9 }\end{array}$ & 9 & CAPP & 30 & 3 & 6 & 9 & 2 & 1 & $D$ & E & 2 & 51 \\
\hline 63 & $48 / \mathrm{M}$ & $\begin{array}{l}\mathrm{L} 50 \\
\mathrm{~S} 1\end{array}$ & 10 & CAPP & 15 & 2 & 2 & 8 & 2 & 1 & $E$ & $\mathrm{E}$ & 2 & 38 \\
\hline 64 & $28 / \mathrm{M}$ & $\begin{array}{l}\mathrm{L} 2 \square \\
\mathrm{L} 3\end{array}$ & 15 & CAPP & 20 & 6 & 7 & 9 & 2 & 1 & $D$ & E & 1 & 40 \\
\hline 65 & $49 / \mathrm{M}$ & L2 & 15 & AP & 15 & 3 & 3 & 9 & 2 & 1 & $E$ & E & 1 & 49 \\
\hline 66 & $43 / F$ & $\begin{array}{l}\mathrm{L} 10 \\
\mathrm{~L} 2\end{array}$ & 10 & AP & 13 & 3 & 4 & 7 & 1 & 0 & $E$ & E & 2 & 50 \\
\hline 67 & $49 / \mathrm{M}$ & T10 & 9 & $\mathrm{AP}$ & 16 & 6 & 6 & 7 & 1 & 0 & $D$ & $E$ & 1 & 50 \\
\hline 68 & $51 / F$ & $\begin{array}{l}\text { L4 } \\
\text { L5 }\end{array}$ & 3 & CAPP & 19 & 5 & 5 & 7 & 1 & 0 & $E$ & E & 2 & 57 \\
\hline 69 & $34 / F$ & $\begin{array}{l}\text { L10 } \\
\text { L2 }\end{array}$ & 12 & AP & 15 & 6 & 6 & 9 & 1 & 1 & $E$ & $E$ & 1 & 39 \\
\hline 70 & $21 / F$ & $\begin{array}{l}\text { L2ロ } \\
\text { L3 }\end{array}$ & 11 & CAPP & 26 & 3 & 4 & 7 & 1 & 0 & $E$ & E & 1 & 50 \\
\hline 71 & $59 / \mathrm{M}$ & $\begin{array}{l}\mathrm{L} 50 \\
\mathrm{~S} 1\end{array}$ & 14 & CAPP & 19 & 5 & 6 & 7 & 2 & 1 & $E$ & $\mathrm{E}$ & 2 & 42 \\
\hline 72 & $42 / F$ & $\begin{array}{l}\text { T110 } \\
\text { T12 }\end{array}$ & 15 & AP & 15 & 5 & 5 & 8 & 2 & 1 & $D$ & E & 1 & 48 \\
\hline
\end{tabular}




\begin{tabular}{|c|c|c|c|c|c|c|c|c|c|c|c|c|c|c|}
\hline \multirow{2}{*}{$\begin{array}{l}\text { Case } \\
\text { No. }\end{array}$} & \multirow{2}{*}{$\begin{array}{l}\text { Age(Y) } \\
\text { /Sex }\end{array}$} & \multirow[t]{2}{*}{ Level } & \multirow{2}{*}{$\begin{array}{l}\text { Duration of } \\
\text { disease(mo) }\end{array}$} & \multirow{2}{*}{$\begin{array}{l}\text { Surgical } \\
\text { procedure }\end{array}$} & \multicolumn{3}{|c|}{ Kyphotic angle } & \multicolumn{3}{|c|}{ VAS pain score } & \multicolumn{2}{|c|}{$\begin{array}{l}\text { Neurologic } \\
\text { status(ASIA) }\end{array}$} & \multirow{2}{*}{$\begin{array}{l}\text { Fusion } \\
\text { grade }\end{array}$} & \multirow{2}{*}{$\begin{array}{l}\text { Follow- } \\
\text { up } \\
\text { (months) }\end{array}$} \\
\hline & & & & & $\begin{array}{l}\text { Pre- } \\
\text { op }\end{array}$ & $\begin{array}{l}\text { Post- } \\
\text { op }\end{array}$ & FFU & $\begin{array}{l}\text { Pre- } \\
\text { op }\end{array}$ & $\begin{array}{l}\text { Post- } \\
\text { op }\end{array}$ & FFU & $\begin{array}{l}\text { Pre- } \\
\text { op }\end{array}$ & FFU & & \\
\hline 73 & $49 / \mathrm{M}$ & $\begin{array}{l}\mathrm{L} 4 \sim \\
\mathrm{S} 1\end{array}$ & 8 & CAPP & 21 & 2 & 5 & 8 & 1 & 1 & E & E & 2 & 57 \\
\hline 74 & $41 / M$ & $\begin{array}{l}\text { L3ロ } \\
\text { L4 }\end{array}$ & 10 & CAPP & 25 & 5 & 5 & 7 & 1 & 0 & E & E & 1 & 53 \\
\hline 75 & $61 / F$ & $\begin{array}{l}\mathrm{L} 10 \\
\mathrm{~L} 2\end{array}$ & 11 & AP & 16 & 4 & 6 & 7 & 1 & 0 & E & E & 2 & 54 \\
\hline 76 & $56 / F$ & $\begin{array}{l}\mathrm{L} 3 \square \\
\mathrm{L} 4\end{array}$ & 8 & CAPP & 22 & 4 & 4 & 7 & 1 & 0 & E & E & 1 & 44 \\
\hline 77 & $34 / \mathrm{M}$ & $\begin{array}{l}\mathrm{L} 4 \square \\
\mathrm{L} 5\end{array}$ & 4 & CAPP & 16 & 4 & 5 & 9 & 1 & 1 & E & E & 1 & 38 \\
\hline 78 & $54 / F$ & $\begin{array}{l}\mathrm{L} 2 \square \\
\mathrm{L} 3\end{array}$ & 13 & CAPP & 27 & 5 & 6 & 9 & 2 & 1 & E & E & 2 & 41 \\
\hline 79 & $21 / F$ & L3 & 15 & AP & 14 & 4 & 4 & 7 & 1 & 0 & E & E & 1 & 39 \\
\hline 80 & $39 / \mathrm{M}$ & $\begin{array}{l}\text { T110 } \\
\text { T12 }\end{array}$ & 5 & AP & 13 & 3 & 3 & 9 & 2 & 1 & D & E & 2 & 36 \\
\hline 81 & $47 / F$ & $\begin{array}{l}\mathrm{L} 10 \\
\mathrm{~L} 2\end{array}$ & 6 & AP & 22 & 2 & 4 & 7 & 1 & 0 & E & $\mathrm{E}$ & 1 & 46 \\
\hline 82 & $53 / \mathrm{M}$ & $\begin{array}{l}\mathrm{L} 4 \square \\
\mathrm{L} 5\end{array}$ & 5 & CAPP & 30 & 5 & 5 & 7 & 2 & 1 & E & $\mathrm{E}$ & 2 & 48 \\
\hline 83 & $45 / F$ & L5 & 7 & CAPP & 24 & 3 & 3 & 8 & 3 & 1 & E & $\mathrm{E}$ & 1 & 53 \\
\hline 84 & $23 / F$ & L4 & 6 & AP & 17 & 3 & 4 & 9 & 3 & 2 & $E$ & $\mathrm{E}$ & 1 & 52 \\
\hline 85 & $39 / \mathrm{M}$ & $\begin{array}{l}\text { L10 } \\
\text { L2 }\end{array}$ & 15 & AP & 15 & 5 & 5 & 8 & 1 & 0 & $E$ & $\mathrm{E}$ & 1 & 48 \\
\hline 86 & $32 / \mathrm{M}$ & $\begin{array}{l}\text { L3a } \\
\text { L4 }\end{array}$ & 12 & CAPP & 27 & 6 & 7 & 9 & 1 & 0 & E & $\mathrm{E}$ & 1 & 37 \\
\hline 87 & $54 / \mathrm{M}$ & $\begin{array}{l}\mathrm{L} 4 \square \\
\mathrm{L} 5\end{array}$ & 14 & CAPP & 22 & 2 & 3 & 7 & 1 & 0 & E & $\mathrm{E}$ & 2 & 39 \\
\hline 88 & $61 / F$ & $\begin{array}{l}\text { T3! } \\
\text { T4 }\end{array}$ & 10 & CAPP & 27 & 4 & 4 & 9 & 3 & 1 & B & B & 2 & 53 \\
\hline 89 & $56 / F$ & $\begin{array}{l}\text { T110 } \\
\text { T12 }\end{array}$ & 15 & CAPP & 23 & 6 & 6 & 9 & 2 & 1 & C & D & 2 & 55 \\
\hline 90 & $21 / F$ & L5 & 11 & CAPP & 19 & 3 & 4 & 7 & 1 & 0 & $E$ & $\mathrm{E}$ & 1 & 54 \\
\hline 91 & $46 / \mathrm{M}$ & $\begin{array}{l}\text { T3 } \\
\text { T5 }\end{array}$ & 5 & CAPP & 30 & 2 & 4 & 9 & 1 & 0 & D & $\mathrm{E}$ & 2 & 40 \\
\hline 92 & $66 / F$ & $\begin{array}{l}\text { T91 } \\
\text { T10 }\end{array}$ & 15 & $\mathrm{AP}$ & 20 & 5 & 5 & 7 & 1 & 0 & D & $\mathrm{E}$ & 2 & 52 \\
\hline 93 & $56 / \mathrm{M}$ & $\begin{array}{l}\mathrm{L} 4 \square \\
5 \mathrm{~L}\end{array}$ & 4 & CAPP & 17 & 3 & 3 & 8 & 1 & 1 & E & E & 2 & 47 \\
\hline 94 & $36 / \mathrm{M}$ & $\begin{array}{l}\mathrm{L} 10 \\
2 \mathrm{~L}\end{array}$ & 15 & AP & 18 & 2 & 4 & 7 & 2 & 0 & $E$ & $\mathrm{E}$ & 1 & 44 \\
\hline 95 & $58 / F$ & $\begin{array}{l}\text { T10 } \\
\text { T2 }\end{array}$ & 6 & AP & 21 & 6 & 6 & 8 & 1 & 0 & B & B & 2 & 44 \\
\hline 96 & $53 \mathrm{~F}$ & $\begin{array}{l}\text { T8ם } \\
\text { T9 }\end{array}$ & 3 & AP & 22 & 4 & 4 & 9 & 1 & 1 & D & $E$ & 1 & 44 \\
\hline 97 & $63 / \mathrm{M}$ & $\begin{array}{l}\text { T7ロ } \\
\text { T8 }\end{array}$ & 3 & AP & 12 & 2 & 3 & 9 & 1 & 1 & D & E & 2 & 54 \\
\hline
\end{tabular}




\begin{tabular}{|c|c|c|c|c|c|c|c|c|c|c|c|c|c|c|}
\hline \multirow{2}{*}{$\begin{array}{l}\text { Case } \\
\text { No. }\end{array}$} & \multirow{2}{*}{$\begin{array}{l}\operatorname{Age}(Y) \\
\text { /Sex }\end{array}$} & \multirow[t]{2}{*}{ Level } & \multirow{2}{*}{$\begin{array}{l}\text { Duration of } \\
\text { disease(mo) }\end{array}$} & \multirow{2}{*}{$\begin{array}{l}\text { Surgical } \\
\text { procedure }\end{array}$} & \multicolumn{3}{|c|}{ Kyphotic angle } & \multicolumn{3}{|c|}{ VAS pain score } & \multicolumn{2}{|c|}{$\begin{array}{l}\text { Neurologic } \\
\text { status(ASIA) }\end{array}$} & \multirow{2}{*}{$\begin{array}{l}\text { Fusion } \\
\text { grade }\end{array}$} & \multirow{2}{*}{$\begin{array}{l}\text { Follow- } \\
\text { up } \\
\text { (months) }\end{array}$} \\
\hline & & & & & $\begin{array}{l}\text { Pre- } \\
\text { op }\end{array}$ & $\begin{array}{l}\text { Post- } \\
\text { op }\end{array}$ & FFU & $\begin{array}{l}\text { Pre- } \\
\text { op }\end{array}$ & $\begin{array}{l}\text { Post- } \\
\text { op }\end{array}$ & FFU & $\begin{array}{l}\text { Pre- } \\
\text { op }\end{array}$ & FFU & & \\
\hline 98 & $41 / \mathrm{M}$ & $\begin{array}{l}\text { T2 } \\
\text { T4 }\end{array}$ & 9 & CAPP & 23 & 5 & 5 & 8 & 1 & 1 & B & C & 2 & 54 \\
\hline 99 & $53 / F$ & $\begin{array}{l}\text { T12 } \\
\mathrm{L} 1\end{array}$ & 9 & CAPP & 26 & 3 & 4 & 9 & 2 & 1 & D & E & 2 & 55 \\
\hline 100 & $29 / \mathrm{M}$ & $\begin{array}{l}\mathrm{L} 5 \square \\
\text { S1 }\end{array}$ & 14 & CAPP & 19 & 3 & 4 & 8 & 2 & 1 & E & E & 1 & 37 \\
\hline 101 & $43 / \mathrm{M}$ & $\begin{array}{l}\text { L4 } \\
\text { L5 }\end{array}$ & 4 & CAPP & 23 & 5 & 5 & 7 & 1 & 0 & E & E & 1 & 50 \\
\hline 102 & $56 / \mathrm{M}$ & $\begin{array}{l}\text { L3! } \\
\text { L4 }\end{array}$ & 4 & CAPP & 19 & 2 & 3 & 7 & 1 & 0 & E & E & 2 & 39 \\
\hline 103 & $44 / F$ & $\begin{array}{l}\text { T8 } \\
\text { T10 }\end{array}$ & 14 & CAPP & 29 & 2 & 4 & 8 & 1 & 0 & C & D & 2 & 38 \\
\hline 104 & $47 / \mathrm{M}$ & $\begin{array}{l}\text { L4 } \\
\text { L5 }\end{array}$ & 14 & CAPP & 17 & 5 & 5 & 9 & 3 & 2 & E & E & 2 & 42 \\
\hline 105 & $48 / \mathrm{M}$ & $\begin{array}{l}\text { L2! } \\
\text { L3 }\end{array}$ & 14 & CAPP & 28 & 4 & 6 & 8 & 2 & 1 & E & E & 2 & 52 \\
\hline 106 & $56 / F$ & $\begin{array}{l}\text { L3! } \\
\text { L4 }\end{array}$ & 15 & CAPP & 21 & 2 & 4 & 7 & 1 & 0 & E & E & 2 & 56 \\
\hline 107 & $54 / F$ & $\begin{array}{l}\text { T90 } \\
\text { T10 }\end{array}$ & 6 & AP & 20 & 3 & 4 & 7 & 1 & 1 & E & E & 2 & 36 \\
\hline 108 & $56 / F$ & $\begin{array}{l}\mathrm{L} 3 \square \\
\mathrm{L} 4\end{array}$ & 5 & CAPP & 25 & 6 & 6 & 7 & 1 & 0 & E & E & 1 & 45 \\
\hline 109 & $53 / \mathrm{M}$ & $\begin{array}{l}\text { L2! } \\
\text { L3 }\end{array}$ & 5 & AP & 19 & 5 & 6 & 7 & 2 & 1 & E & $E$ & 1 & 49 \\
\hline 110 & $55 / F$ & $\begin{array}{l}\text { L4] } \\
\text { L5 }\end{array}$ & 14 & CAPP & 15 & 2 & 2 & 9 & 3 & 1 & E & E & 1 & 57 \\
\hline 111 & $66 / F$ & $\begin{array}{l}\text { T12口 } \\
\text { L1 }\end{array}$ & 13 & AP & 22 & 6 & 8 & 8 & 1 & 0 & D & $E$ & 2 & 53 \\
\hline 112 & $52 / \mathrm{M}$ & $\begin{array}{l}\text { L2" } \\
\text { L3 }\end{array}$ & 6 & CAPP & 20 & 4 & 4 & 8 & 1 & 0 & E & E & 1 & 39 \\
\hline 113 & $57 / F$ & $\begin{array}{l}\text { T91 } \\
\text { T10 }\end{array}$ & 12 & AP & 18 & 3 & 4 & 7 & 1 & 0 & D & $\mathrm{E}$ & 2 & 50 \\
\hline 114 & $60 / F$ & $\begin{array}{l}\text { L3ם } \\
\text { L5 }\end{array}$ & 11 & CAPP & 22 & 5 & 6 & 7 & 1 & 0 & E & E & 2 & 50 \\
\hline 115 & $28 / \mathrm{M}$ & $\begin{array}{l}\text { T70 } \\
\text { T8 }\end{array}$ & 6 & CAPP & 23 & 4 & 4 & 8 & 1 & 0 & C & D & 1 & 47 \\
\hline 116 & $23 / \mathrm{M}$ & $\begin{array}{l}\text { L2" } \\
\text { L3 }\end{array}$ & 3 & CAPP & 25 & 2 & 3 & 9 & 2 & 1 & E & E & 2 & 40 \\
\hline 117 & $55 / F$ & $\begin{array}{l}\text { T10! } \\
\text { T11 }\end{array}$ & 7 & AP & 12 & 2 & 3 & 7 & 1 & 0 & D & E & 1 & 52 \\
\hline 118 & $60 / \mathrm{M}$ & $\begin{array}{l}\text { T8D } \\
\text { T9 }\end{array}$ & 7 & AP & 16 & 5 & 6 & 9 & 2 & 2 & D & E & 2 & 38 \\
\hline 119 & $21 / F$ & $\begin{array}{l}\text { T12 } \\
\sim \mathrm{L3}\end{array}$ & 9 & CAPP & 25 & 6 & 7 & 6 & 1 & 0 & D & E & 2 & 54 \\
\hline 120 & $68 / F$ & $\begin{array}{l}\text { L2] } \\
\text { L3 }\end{array}$ & 8 & CAPP & 23 & 2 & 2 & 7 & 1 & 1 & E & E & 3 & 54 \\
\hline
\end{tabular}




\begin{tabular}{|c|c|c|c|c|c|c|c|c|c|c|c|c|c|c|}
\hline \multirow{2}{*}{$\begin{array}{l}\text { Case } \\
\text { No. }\end{array}$} & \multirow{2}{*}{$\begin{array}{l}\text { Age(Y) } \\
\text { /Sex }\end{array}$} & \multirow[t]{2}{*}{ Level } & \multirow{2}{*}{$\begin{array}{l}\text { Duration of } \\
\text { disease(mo) }\end{array}$} & \multirow{2}{*}{$\begin{array}{l}\text { Surgical } \\
\text { procedure }\end{array}$} & \multicolumn{3}{|c|}{ Kyphotic angle } & \multicolumn{3}{|c|}{ VAS pain score } & \multicolumn{2}{|c|}{$\begin{array}{l}\text { Neurologic } \\
\text { status(ASIA) }\end{array}$} & \multirow{2}{*}{$\begin{array}{l}\text { Fusion } \\
\text { grade }\end{array}$} & \multirow{2}{*}{$\begin{array}{l}\text { Follow- } \\
\text { up } \\
\text { (months) }\end{array}$} \\
\hline & & & & & $\begin{array}{l}\text { Pre- } \\
\text { op }\end{array}$ & $\begin{array}{l}\text { Post- } \\
\text { op }\end{array}$ & FFU & $\begin{array}{l}\text { Pre- } \\
\text { op }\end{array}$ & $\begin{array}{l}\text { Post- } \\
\text { op }\end{array}$ & FFU & $\begin{array}{l}\text { Pre- } \\
\text { op }\end{array}$ & FFU & & \\
\hline 121 & $50 / \mathrm{M}$ & $\begin{array}{l}\text { T110 } \\
\text { T12 }\end{array}$ & 10 & AP & 16 & 2 & 4 & 7 & 1 & 0 & E & E & 2 & 49 \\
\hline 122 & $26 / M$ & $\begin{array}{l}\text { T6 } \\
\text { T7 }\end{array}$ & 14 & CAPP & 27 & 5 & 5 & 8 & 2 & 0 & $E$ & E & 1 & 45 \\
\hline 123 & $25 / M$ & $\begin{array}{l}\text { T70 } \\
\text { T8 }\end{array}$ & 15 & CAPP & 24 & 5 & 5 & 8 & 1 & 0 & $\mathrm{D}$ & E & 1 & 57 \\
\hline 124 & $69 / \mathrm{M}$ & $\begin{array}{l}\text { T100 } \\
\text { T11 }\end{array}$ & 12 & AP & 20 & 6 & 6 & 7 & 2 & 0 & C & E & 3 & 56 \\
\hline 125 & $47 / M$ & $\begin{array}{l}\text { T10! } \\
\text { T1 }\end{array}$ & 3 & AP & 15 & 6 & 7 & 8 & 1 & 0 & $\mathrm{D}$ & E & 1 & 43 \\
\hline 126 & $62 / \mathrm{M}$ & $\begin{array}{l}\text { T12口 } \\
\text { L1 }\end{array}$ & 13 & AP & 18 & 5 & 7 & 7 & 1 & 0 & $\mathrm{D}$ & E & 2 & 36 \\
\hline 127 & $49 / \mathrm{M}$ & $\begin{array}{l}\text { L2" } \\
\text { L3 }\end{array}$ & 15 & AP & 18 & 2 & 4 & 8 & 2 & 1 & E & E & 1 & 57 \\
\hline 128 & $44 / \mathrm{M}$ & $\begin{array}{l}\mathrm{L} 4 \square \\
\mathrm{L} 5\end{array}$ & 9 & CAPP & 21 & 3 & 3 & 9 & 3 & 1 & $\mathrm{E}$ & E & 2 & 41 \\
\hline 129 & $59 / \mathrm{M}$ & $\begin{array}{l}\text { T8 } \\
\text { T9 }\end{array}$ & 13 & CAPP & 20 & 4 & 5 & 8 & 1 & 0 & B & B & 2 & 48 \\
\hline 130 & $63 / F$ & L2 & 7 & AP & 14 & 5 & 6 & 9 & 2 & 1 & $E$ & $\mathrm{E}$ & 2 & 56 \\
\hline 131 & $20 / \mathrm{M}$ & $\begin{array}{l}\text { T12 } \\
\sim \text { L2 }\end{array}$ & 7 & CAPP & 22 & 4 & 4 & 9 & 1 & 1 & $E$ & $\mathrm{E}$ & 1 & 46 \\
\hline 132 & $42 / F$ & $\begin{array}{l}\mathrm{L} 3 \square \\
\mathrm{L} 4\end{array}$ & 3 & CAPP & 27 & 3 & 4 & 9 & 2 & 1 & $D$ & E & 2 & 49 \\
\hline 133 & $54 / \mathrm{M}$ & $\begin{array}{l}\text { T8 } \\
\text { T10 }\end{array}$ & 4 & CAPP & 29 & 4 & 5 & 7 & 1 & 0 & $E$ & E & 2 & 36 \\
\hline
\end{tabular}

There were 276 involved vertebral bodies, with a single level involvement in 13 patients, two levels in 102 patients, and three levels or more in 18 patients. Patients with thoracic $(n=27)$, thoracolumbar $(n=36)$, and lumbar $(n=70)$ involvement were comparable in regards to kyphosis angle and deformity correction. Patients with neural deficits were graded according to the ASIA scale [15]. 44 of the 132 patients (33.3\%), 4 ASIA B, 10 ASIA C, and 30 ASIA D. The diagnostic characteristics and clinical outcome of patients were listed in the Table 1.

Preoperatively, all patients were evaluated with radiographic examination and computed tomography (CT) and most of the patients were evaluated magnetic resonance imaging (MRI). Radiographic examination revealed vertebral destruction/collapse, kyphotic deformities and paravertebral abscess. CT scans mostly revealed the extent of bony destruction and location of abscess. MRI revealed vertebral destruction, collapse, paravertebral and/or psoas abscess, and spinal cord compression. The kyphosis deformity was measured on lateral radiographs according the angle between the superior endplate of the first upper uninvolved vertebra and the first lower uninvolved vertebra. In the lumbosacral region, the kyphosis angle was measured by two lines along the posterior border of the first normal vertebra above the level of the lesion and posterior margin of sacrum on lateral radiographs.

All patients underwent antituberculous chemotherapy (Rifampicin, $450 \mathrm{mg} / \mathrm{d}$; Isoniazid, $300 \mathrm{mg} / \mathrm{d}$; Ethambutol $750 \mathrm{mg} / \mathrm{d}$ )) before surgery for 3 weeks, except those who need urgent decompression for recently developed progressive neurologic deficits. All procedures were preformed by the same surgeon (YM.L). A transpleural or retroperitoneal approach was used according to the location of infection. Perioperatively, frozen-section histopathological examination was performed to confirm the diagnose of tuberculosis, according to the presence of Langerhans giant cells, granuloma, and caseating necrosis on the histopathological sections. After radical debridement and decompression, we used titanium cages filled with crushed cancellous allograft for anterior column reconstruction. Structural stability was secured with the anterior instrumentation when one vertebra has been removed or posterior instrumentation when two or more vertebrae have been removed resulted from radical debridement. Postoperatively, patients were mobilized for six weeks with the aid of a molded Boston type brace. Postoperatively, histopathological examination and cultures of material obtained from the debridement confirmed the diagnosis of 
tuberculosis. All patients received antituberculous chemotherapy up to 12 months postoperatively. Liver and renal functions were monitored regularly throughout this period. Erythrocyte sedimentation rate (ESR) and C-reactive protein (CRP) were also monitored regularly to exclude the presence of infection activity.

Anteroposterior (AP) and lateral radiographs were taken immediately after surgery, 3 months, 6 months, and 12 months postoperatively, and subsequently at yearly intervals. All radiographs were evaluated by the same observer (X.W), who was blinded to the clinical outcome. The presence of allograft incorporation was determined by plain radiographs and was further verified by CT scans in some patients.

Clinical evaluation was done by another observer (HC.C), who was blinded to the radiographic outcome. The patients' preoperative and postoperative neurologic status was evaluated and documented according to the American Spinal Injury Association (ASIA) Impairment Scale (AIS) [15]. Clinical evaluation of back pain was evaluated and documented according to VAS pain score [16].

Descriptive statistics were used for all variables with distributions assessed for normality. The paired $t$-tests were conducted to compare preoperative and post-operative changes of kyphosis and VAS pain score. The Statistical Package for the Social Sciences (version 17.0 for Windows; SPSS Inc, Chicago, IL) was used for statistical analysis. The difference was considered to be statistically significant at the $p<0.05$ level.

\section{Results}

The average operating time was 2.4 hours (range, 1.9 to 4.1 hours) for anterior surgeries, and 5.3 hours (range, 4.2 to 7.1 hours) for combined anterior and posterior surgeries. The average perioperative blood loss was $700 \mathrm{ml}$ (range, 471 to $1193 \mathrm{ml}$ ) for anterior surgeries, and $1145 \mathrm{ml}$ (range, 864 to $1393 \mathrm{ml}$ ) for combined anterior and posterior surgeries. The average postoperative hospitalization was 16.8 days (range, 12 to 21 days).

The preoperative kyphosis deformity improved in all patients postoperatively from a mean of $20.8^{\circ}\left(\right.$ range, $\left.12^{\circ}-35^{\circ}\right)$ to $3.9^{\circ}\left(\right.$ range, $\left.2^{\circ}-7^{\circ}\right)$, with the average deformity correction of $16.1^{\circ}(t=38.59, p<0.0001)$. At the final follow-up, the average kyphosis was $4.7^{\circ}\left(\right.$ range, $\left.2^{\circ}-9^{\circ}\right)$, which corresponded to a mean angular loss of correction (increase in kyphosis) of $0.8^{\circ}(t=-11.1, p<0.0001)$.

The earliest radiologic evidence of allograft incorporation was identified between 18 and 24 months after surgery, and bony fusion was seen in all patients at final follow-up. Based on the criteria of Bridwell et al [17], we put forward our criteria to classify allograft incorporation at the last follow-up. Grade 1 (Fig. 1) fusion with healing of compromised vertebra (49 patients); Grade 2 (Fig. 2) graft intact with no lucencies at the top or bottom, but without full healing of compromised vertebra (73 patients); Grade 3 (Fig. 3) fusion, graft intact but definite lucency at the top or bottom of the graft (11 patients); Grade 4 not fusion with resorption of bone graft or collapse (no patient).

Back pain greatly relieved in all the patients. The average VAS pain score was 7.7 (range, 6-9) preoperatively, and decreased to 1.4 (range, 1$3) 3$ months after surgery $(t=97.88, p<0.0001$ vs. initial). At final follow-up, the average VAS pain score was 0.5 (range, $0-2$ ).

Of the 44 patients who developed neural deficits before their operations, 41 patients improved according to the ASIA AIS. Three patients did not have substantial improvement and remained Grade AIS B.

There were no major complications, such as implant failure, wound infection and iatrogenic neurovascular injuries. Infection control was achieved in all patients except for tuberculosis recurrence in one patient.

\section{Discussion}

The present study suggests that radical debridement followed by allograft with titanium cage in reconstruction of the anterior spinal column offers very satisfactory results in patients with tuberculous spondylitis who need for surgical intervention. It provides immediate sagittal stability, offers lasting kyphosis correction, bypasses the donor site morbidity and difficulties involved in obtaining structural autograft.

Early initiation of the proper antituberculous chemotherapy coupled with immobilization leads to a favorable outcome in most patients with spinal tuberculosis [5]. Failure of nonsurgical treatment, abscess formation, neurological deterioration and progressive kyphotic deformity or instability are all indications for surgical intervention $[2,6]$. Goals of surgical intervention for spinal tuberculosis include alleviation of pain, spinal cord decompression, prevention and correction of deformity with spinal stabilization, and resection of the infectious paraspinal component to allow antituberculous chemotherapy to work more effectively [18].

Anterior radical debridement and fusion has been advocated for the treatment of patients with tuberculous spondylitis since 1960 [13, 19, 20]. Surgical debridement of the compromised anterior column results in a further loss of structural integrity that requires anterior reconstruction. Structural autograft continues to be the historical standard in anterior reconstruction [21]. However, there may be substantial morbidity

Page $10 / 16$ 
associated with the harvest of large structural autografts, such as infection, pain, and loss of structural support at the harvest site, in addition to the limited supply of structural autograft that makes it difficult or impractical in many patients with tuberculous spondylitis who require surgical treatment [22]. Other options available for anterior column reconstruction include structural allograft or titanium cages filled with crushed cancellous allograft and autograft $[14,23]$. Titanium cages have been shown to be effective for anterior column reconstruction of spine in traumatic, pathologic and infection [23-26]. The titanium cage can be filled with autograft in addition to crushed cancellous allograft that can allow rapid incorporation of the graft. The cage with bone grafts allows for a more solid fixation construct and minimizes the risk of graft dislodgement or subsidence that are well documented complications when structural bone graft alone is used [11, 12, 27-29]. It also provides safer stability than structural bone graft alone in the infection region that allows tissue rest and earlier mobilization [12, 27]. Our consideration for using titanium cages filled with crushed cancellous allograft/and autograft was to overcome the morbidity associated with structural autograft and the slow rate of graft incorporation associated with structural allograft. This study identified a correction of kyphosis from preoperative $20.8^{\circ}$ to immediate postoperative $3.9^{\circ}$, and a final kyphosis of $4.7^{\circ}$, which corresponded to a mean loss of correction of $0.8^{\circ}$, resulted from subsidence of titanium cage. This has shown that the titanium cage is effective in maintaining sagittal alignment over a long postoperative period.

The criteria described by Bridwell et al is probably the gold standard in assessing the completeness of bony fusion for the high intraobserver and interobserver agreement $[17,30]$. As the Bridwell criteria were initially described to identify bony fusion in cases of strut allograft rather than titanium cages, we modified it in this study considering that the achievement of bony healing of compromised vertebra despite the presence of remodeling may be the more important factor for the ultimate outcome of spinal tuberculosis. The duration of biologic incorporation of allografts is of clinical importance because it may be slow and unpredictable, resulted from immunological responses to donor antigens [31, 32]. This study identified a Grade 1 or 2 allograft incorporation in $92 \%$ of the patients, suggesting that immunological responses does not hinder the development of solid bony fusion, and that the achievement of this solid bony fusion despite the duration of allograft incorporation may be the more important factor for the ultimate outcome of spinal tuberculosis.

Implantation of metallic instrumentation into the infected region, however, is a matter of debate because although implants help provide structural stability, the presence of foreign material in an area of mycobacterial infection may interfere with successful eradication of infection $[12,27,28]$. There were experimental studies suggest that Mycobacterium tuberculosis, unlike pyogenic bacteria, has low adherence to metal and forms less polysaccharide biofilm $[33,34]$. Therefore, the use of metal implants in the region of mycobacterial infection may be relatively safe [9]. This study identified tuberculosis recurrence only in one of 133 patients, suggesting that implantation of titanium cages into the region of mycobacterial infection have lower infection risk. Furthermore, we believe that the reconstruction of unstable spinal segments offered by this method may resulted in a more favorable environment for control of infection, graft incorporation, and solid bony fusion.

Although the anterior instrumentation offers more reliable restoration of structural stability compared with posterior instrumentation [35], in patients with two or more severe vertebral destruction that may lead to significant kyphotic deformity [36], either the anterior or posterior instrumentation alone may be insufficient theoretically, and a combined anterior and posterior instrumentation should be more reliable in preventing the development of kyphotic deformity [20,37,38]. There were researches suggest that when two or more vertebrae have been removed resulted from radical debridement, the grafts are prone to failure or resorption [12], and supplemental posterior fusion and instrumentation is an reinforcement to anterior instrumentation and helpful to arrest the infection, correct the kyphosis, prevent progression of kyphosis and promote early fusion [37-39]. Furthermore, the surgical dissection should be wider to fix the anterior plate to the adjacent unaffected vertebrae, which may lead to more complications or infection spread. In the current series, about 60 percent of the patients (80 of 133) had destruction of two or more vertebral bodies. And combined anterior and posterior procedure was performed for these patients. This study identified a correction of kyphosis in these patients from preoperative $23.3^{\circ}$ to immediate postoperative $3.9^{\circ}$, and a final kyphosis of $4.5^{\circ}$, which corresponded to a mean loss of correction of $0.8^{\circ}$, resulted from subsidence of titanium cage. This has shown that although the combined anterior and posterior procedure was related with prolonged operating time, more blood loss, and increased postoperative complications [40], posterior stabilization with instrumentation after anterior spinal debridement and fusion is effective in maintaining sagittal alignment over a long postoperative period.

Our study is mainly limited by the retrospective study and the absence of a control group. As the result of infrequent condition of spinal tuberculosis, it is nearly impossible to conduct a prospective study with a rational timepoint for completion. The fact that all patients were operated on by the same surgeon and were observed for a mean > 3 years by two blinded independent observers may allow us to draw meaningful conclusions.

\section{Conclusion}

Radical debridement followed by allograft with titanium cage in reconstruction of the anterior spinal column offers very satisfactory results in patients with tuberculous spondylitis. It provides immediate stability, offers lasting kyphosis correction, bypasses the donor site morbidity and difficulties involved in obtaining structural autograft.

Page $11 / 16$ 


\section{Declarations}

ACKNOWLEDGEMENTS

Not applicable.

AUTHORS' CONTRIBUTIONS

YJW conceived and designed the study, analyzed the data and wrote

\section{FUNDING}

Not applicable

\section{AVAILABILITY OF DATA AND MATERIALS}

The datasets used and/or analysed during the current study are available from the corresponding author on reasonable request.

\section{ETHICS APPROVAL AND CONSENT TO PARTICIPATE}

Not applicable

\section{CONSENT FOR PUBLICATION}

Written informed consent for publication was obtained from all participants

\section{COMPETING INTERESTS}

The authors declare that they have no competing interests.

\section{References}

1. Ilgazli A, Boyaci H, Basyigit I, Yildiz F. Extrapulmonary tuberculosis: clinical and epidemiologic spectrum of 636 cases. Arch Med Res. 2004;35:435-41.

2. Tuli SM. Tuberculosis of the spine: a historical review. Clin Orthop Relat Res. 2007;460:29-38.

3. Jain AK, Aggarwal A, Mehrotra G. Correlation of canal encroachment with neurological deficit in tuberculosis of the spine. Int Orthop. 1999;23:85-6.

4. Dharmalingam M. Tuberculosis of the spine-the Sabah experience. Epidemiology, treatment and results. Tuberculosis (Edinb). 2004;84:24-8.

5. Chandra SP, Singh A, Goyal N, Laythalling RK, et al. Analysis of changing paradigms of management in 179 patients with spinal tuberculosis over a 12-year period and proposal of a new management algorithm. World Neurosurg. 2013;80:190-203.

6. Krödel A, Stürz H, Siebert CH. Indications for and results of operative treatment of spondylitis and spondylodiscitis. Arch Orthop Trauma Surg. 1991;110:78-82.

7. Li M, Du J, Meng H, Wang Z, Luo Z. One-stage surgical management for thoracic tuberculosis by anterior debridement, decompression and autogenous rib grafts, and instrumentation. Spine J. 2011;11:726-33.

8. Hodgson AR, Stock FE. Anterior spinal fusion: a preliminary communication on the radical treatment of Pott's disease and Pott's paraplegia. Clin Orthop Relat Res. 1994;300:16-23.

9. Benli IT, Kaya A, Acaroğlu E. Anterior instrumentation in tuberculous spondylitis: is it effective and safe? Clin Orthop Relat Res. 2007;460:108-16.

10. Przybylski GJ, Sharan AD. Single-stage autologous bone grafting and internal fixation in the surgical management of pyogenic discitis and vertebral osteomyelitis. J Neurosurg. 2001;94(S1):1-7.

11. Upadhyay SS, Sell P, Saji M, Hsu LC. Surgical management of spinal tuberculosis in adults: Hong Kong operation compared with debridement surgery for short and long term outcome of deformity. Clin Orthop Relat Res. 1994;302:173-82.

12. Yilmaz C, Selek HY, Gürkan I, Erdemli B, Korkusuz Z. Anterior instrumentation for the spinal tuberculosis. J Bone Joint Surg Am. 1999;81:1261-7.

13. Govender S, Parbhoo AH. Support of the anterior column with allografts in tuberculosis of the spine. J Bone Joint Surg Br. 1999;81:1069.

14. Govender S. The outcome of allografts and anterior instrumentation in spinal tuberculosis. Clin Orthop Relat Res. 2002;398:60-6. 
15. American Spinal Injury Association. International standard for neurological and functional classification of spinal injury. Chicago, ASIA 1992.

16. Bodian CA, Freedman G, Hossain S, Eisenkraft JB, Beilin Y. The Visual Analog Scale for Pain: Clinical Significance in Postoperative Patients. Anesthesiology. 2001;95:1356-61.

17. Bridwell KH, Lenke LG, McEmery KW, Baldus C, Blanke K. Anterior fresh frozen structural allografts in the thoracic and lumbar spine. Do they work if combined with posterior fusion and instrumentation in adult patients with kyphosis or anterior column defects? Spine (Phila Pa 1976). 1995; 20:1410-1418.

18. Mehta JS, Bhojraj SY. Tuberculosis of the thoracicspine. A classification based on the selection of surgical strategies. J Bone Joint Surg Br. 2001;83:859-63.

19. Hodgson AR, Stock FE. Anterior spinal fusion for the treatment of tuberculosis of the spine: The operative findings and results of treatment in the first one hundred cases. J Bone Joint Surg Am. 1960;42:295-310.

20. Rajasekaran S, Soundarapandian S. Progression of kyphosis in tuberculosis of the spine, treated by anterior arthrodesis. J Bone Joint Surg Am. 1989;71:1314-23.

21. Krödel A, Krüger A, Lohscheidt K, Pfahler M, Refior HJ. Anterior debridement, fusion, and extrafocal stabilization in the treatment of osteomyelitis of the spine. J Spinal Disord. 1999;12:17-26.

22. Goulet JA, Senunas LE, DeSilva GL, Greenfield ML. Autogenous iliac crest bone graft. Complications and functional assessment. Clin Orthop Relat Res. 1997;339:76-81.

23. Christodoulou AG, Givissis P, Karataglis D, Symeonidis PD, Pournaras J. Treatment of tuberculous spondylitis with anterior stabilization and titanium cage. Clin Orthop Relat Res. 2006;444:60-5.

24. Vaccaro AR, Cirello J. The use of allograft bone and cages in fractures of the cervical, thoracic, and lumbar spine. Clin Orthop Relat Res. 2002;394:19-26.

25. Weigel B, Maghsudi M, Neumann C, Kretschmer R, Müller FJ, Nerlich M. Surgical management of symptomatic spinal metastases. Postoperative outcome and quality of life. Spine (Phila Pa 1976). 1999; 24: 2240-2246.

26. Fayazi AH, Ludwig SC, Dabbah M, Bryan Butler R, Gelb DE. Preliminary results of staged anterior debridement and reconstruction using titanium mesh cages in the treatment of thoracolumbar vertebral osteomyelitis. Spine J. 2004;4:388-95.

27. Faraj AA, Webb JK. Spinal instrumentation for primary pyogenic infection: Report of 31 patients. Acta Orthop Belg. 2000;66:242-7.

28. Safran O, Rand N, Kaplan L, Sagiv S, Floman Y. Sequential or simultaneous, same-day anterior decompression and posterior stabilization in the management of vertebral osteomyelitis in the lumbar spine. Spine (Phila Pa 1976). 1998; 23:1885-1890.

29. Upadhyay SS, Sell P, Saji MJ, Sell B, Yau AC, Leong JC. 17-year prospective study of surgical management of spinal tuberculosis in children: Hong Kong operation combined with debridement surgery for short- and long-term outcome of deformity. Spine (Phila Pa 1976). 1993; 18:1704-1711.

30. Eck KR, Lenke LG, Bridwell KH, Gilula LA, Lashgari CJ, Riew KD. Radiographic assessment of anterior titanium mesh cages. J Spinal Disord. 2000;13:501-10.

31. Goldberg VM, Stevenson S. Natural history of autografts and allografts. Clin Orthop Relat Res. 1987;225:7-16.

32. Stevenson S, Emery SE, Goldberg VM. Factors affecting bone graft incorporation. Clin Orthop Relat Res. 1996;324:66-74.

33. Oga M, Arizono T, Takasita M, Sugioka Y. Evaluation of the risk of instrumentation as a foreign body in spinal tuberculosis: Clinical and biologic study. Spine (Phila Pa 1976). 1993;18:1890-4.

34. Chen WH, Jiang LS, Dai LY. Influence of bacteria on spinal implant-centered infection. Spine (Phila Pa 1976). 2011;36:103-8.

35. Gurr KR, McAfee PC, Shih CM. Biomechanical analysis of anterior and posterior instrumentation systems following corpectomy: A calf spine model. J Bone Joint Surg Am. 1988;70:1182-91.

36. Korkusuz F, Islam C, Korkusuz Z. Prevention of posterior late kyphosis in Pott's disease by anterior decompression and intervertebral grafting. World J Surg. 1997;21:524-8.

37. Chen WJ, Chen $\mathrm{CH}$, Shih $\mathrm{CH}$. Surgical treatment of tuberculous spondylitis: 50 patients followed for 2-8 years. Acta Orthop Scand. 1995;66:137-42.

38. Moon MS, Woo YK, Lee KS, Ha KY, Kim SS, Sun DH. Posterior instrumentation and anterior interbody fusion for tuberculous kyphosis of dorsal and lumbar spines. Spine (Phila Pa 1976). 1995; 20:1910-1916.

39. Chen WJ, Wu CC, Jung CH, Chen LH, Niu CC, Lai PL. Combined anterior and posterior surgeries in the treatment of spinal tuberculous spondylitis. Clin Orthop Relat Res. 2002;398:50-9.

40. Moon MS. Tuberculosis of the spine: Controversies and a new challenge. Spine (Phila Pa 1976). 1997;22:1791-7.

Page $13 / 16$ 
Figures

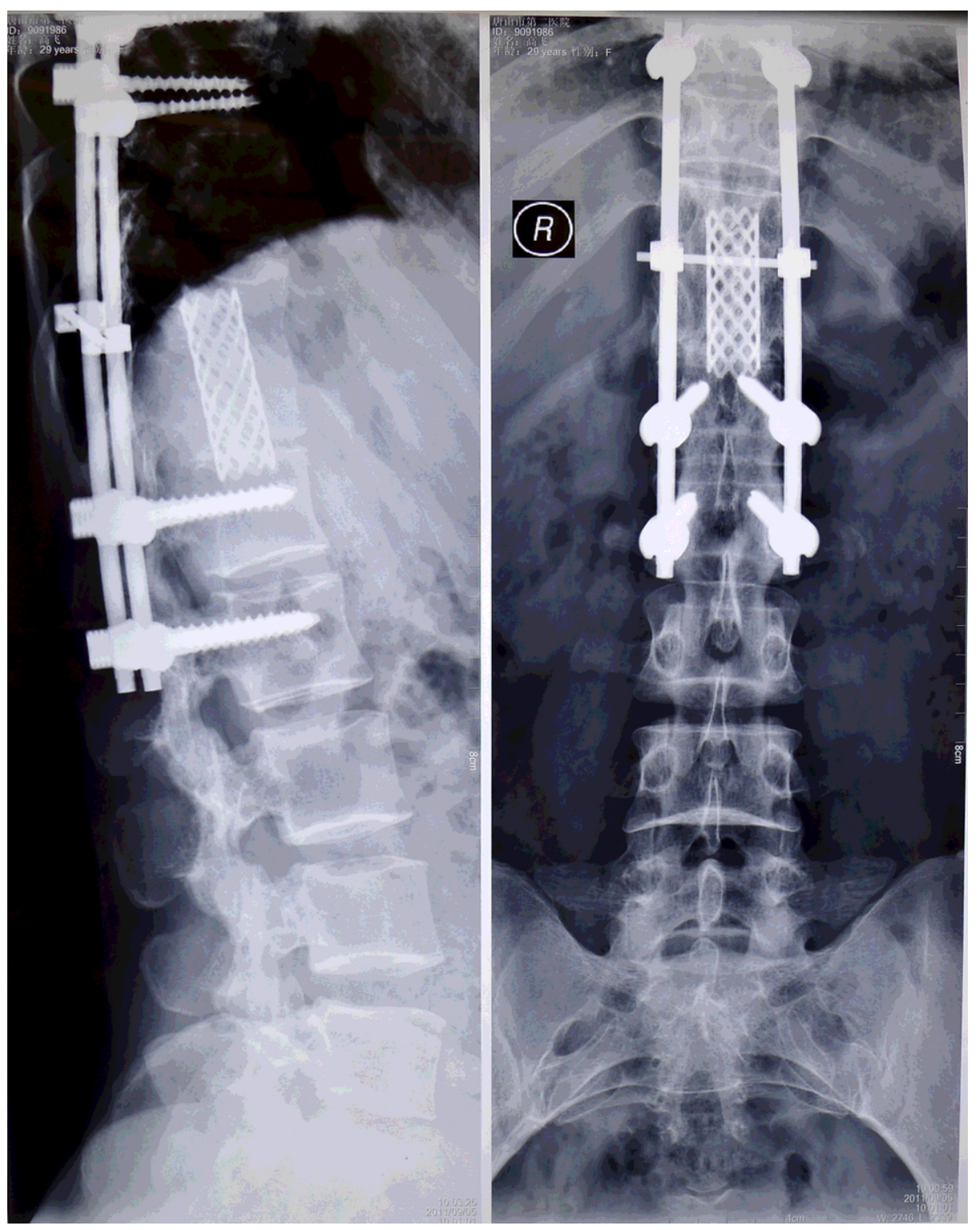

Figure 1

Fusion with healing of compromised vertebra. 


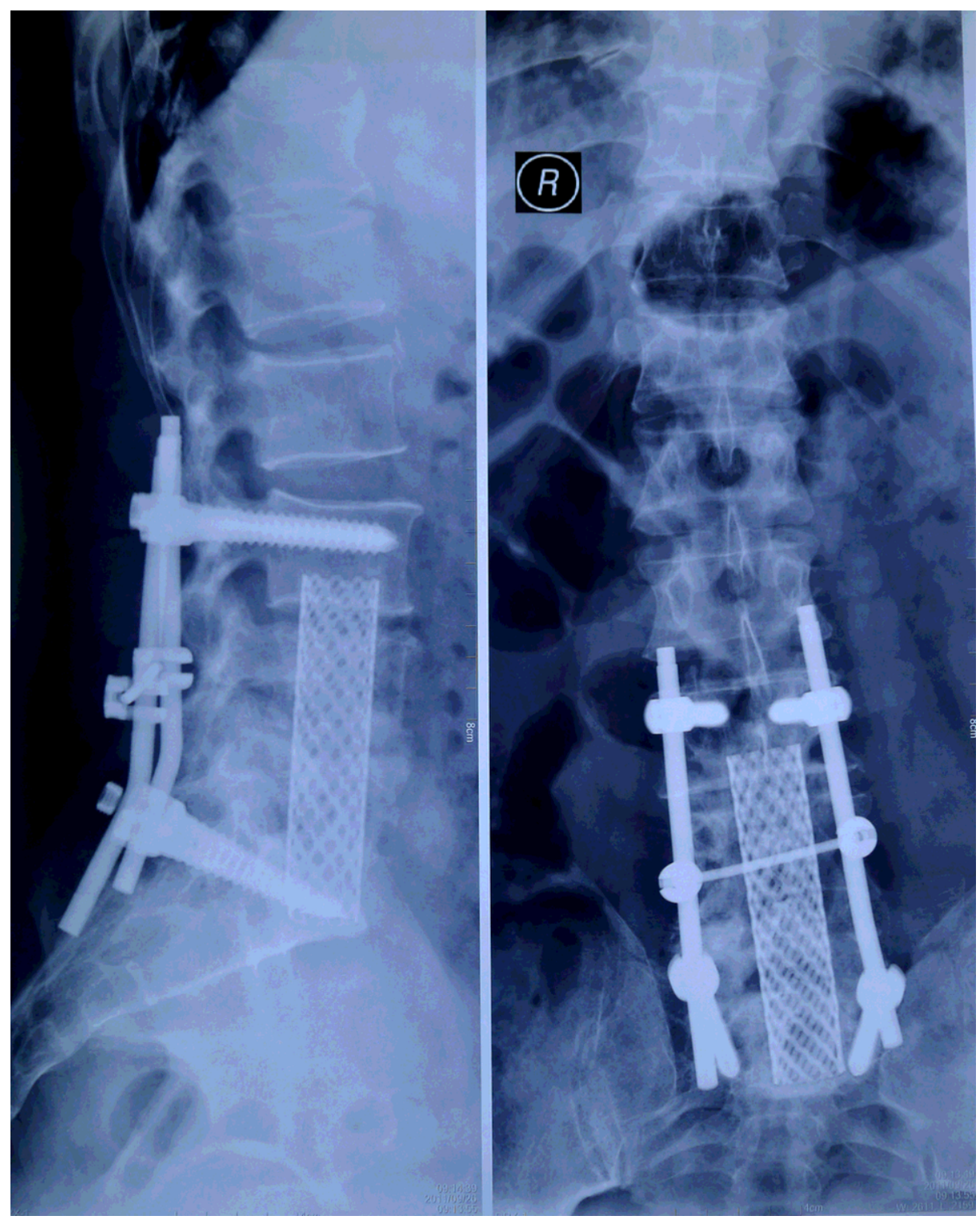

\section{Figure 2}

Graft intact with no lucencies at the top or bottom, but without full healing of compromised vertebra. 


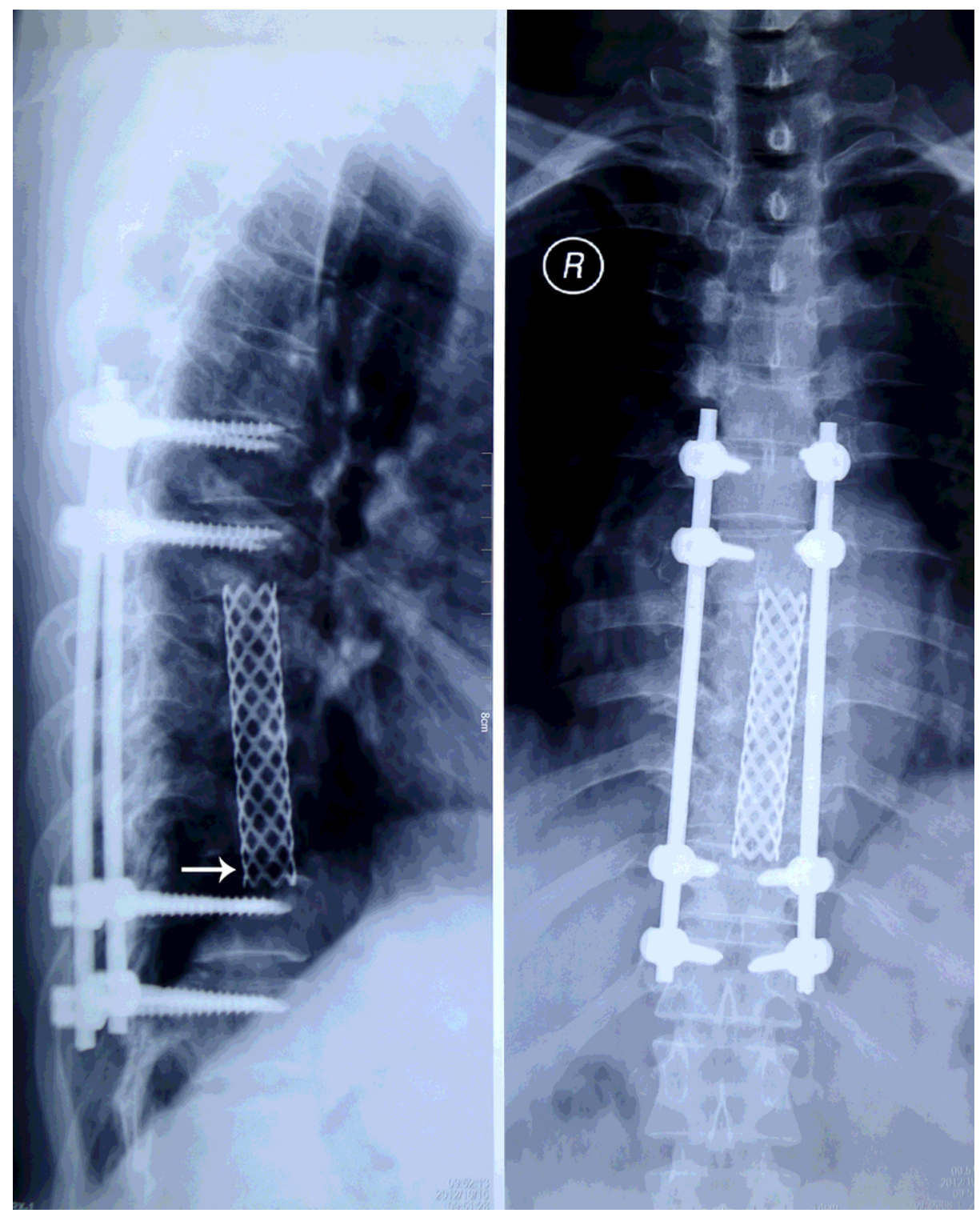

Figure 3

Fusion, graft intact but definite lucency at bottom of the titanium cage. 